\title{
A Quantitative Trait Locus for Chlorophyll Content and its Association with Leaf Photosynthesis in Rice
}

\author{
Toshiyuki Takai • Motohiko Kondo • Masahiro Yano • \\ Toshio Yamamoto
}

Received: 21 May 2010 / Accepted: 9 July 2010 /Published online: 25 July 2010

(C) Springer Science+Business Media, LLC 2010

\begin{abstract}
Leaf photosynthesis, an important determinant of yield potential in rice, can be estimated from measurements of chlorophyll content. We searched for quantitative trait loci (QTLs) for Soil and Plant Analyzer Development (SPAD) value, an index of leaf chlorophyll content, and assessed their association with leaf photosynthesis. QTL analysis derived from a cross between japonica cultivar Sasanishiki and high-yielding indica cultivar Habataki detected a QTL for SPAD value on chromosome 4. This QTL explained 31\% of the total phenotypic variance, and the Habataki allele increased the SPAD value. Chromosomal segment substitution line (CSSL) with the corresponding segment from Habataki had a higher leaf photosynthetic rate and SPAD value than Sasanishiki, suggesting an association between SPAD value and leaf photosynthesis. The CSSL also had a lower specific leaf area (SLA) than Sasanishiki, reflecting its thicker leaves. Substitution mapping under Sasanishiki genetic background demonstrated that QTLs for SPAD value and SLA were co-localized in the 1,798-kb interval. The results suggest that the phenotypes for SPAD value and SLA are controlled by a single locus or two tightly linked loci, and may play an important role in increasing leaf photosynthesis by increasing chlorophyll content or leaf thickness, or both.
\end{abstract}

Keywords Chlorophyll · Leaf area · Photosynthesis .

QTLs $\cdot$ SPAD value $\cdot$ Substitution mapping

T. Takai $\cdot$ M. Kondo

National Institute of Crop Science,

2-1-18, Kannondai,

Tsukuba, Ibaraki 305-8518, Japan

M. Yano $\cdot$ T. Yamamoto $(\bowtie)$

National Institute of Agrobiological Sciences,

2-1-2 Kannondai,

Tsukuba, Ibaraki 305-8602, Japan

e-mail: toshyama@affrc.go.jp

\section{Introduction}

Leaf photosynthesis is the component of canopy photosynthesis that accounts for most of the variation in biomass production and yield (Peng 2000; Yoshida and Horie 2009). While it is still controversial whether increasing leaf photosynthesis increases yield (Evans 1993; Sinclair et al. 2004), recent studies indicate that growth rate around heading stage is critically related with final yield in rice (Takai et al. 2006; Horie et al. 2006), and that new high-yielding rice cultivars, including both inbred and hybrid cultivars, have higher leaf photosynthetic rates than previously released ones, particularly at heading stage (Ohsumi et al. 2007; Peng et al. 2008). To examine this issue, it is necessary to identify genetic factors controlling leaf photosynthesis, and to compare yield potential between donor cultivars and nearisogenic lines (NILs) differing only in leaf photosynthetic ability (Zelitch 1982; Long et al. 2006; Hubbart et al. 2007).

The process of photosynthesis is difficult to measure directly, but a positive relationship between leaf photosynthesis and leaf chlorophyll content has been widely observed in rice (Makino et al. 1983; Kura-Hotta et al. 1987; Xu et al. 1997). Chlorophyll content is generally measured after extraction of chlorophyll from ground leaves with organic solvents (Porra et al. 1989). On the other hand, a digital chlorophyll meter (Soil and Plant Analyzer Development [SPAD] meter) provides a non-destructive method for estimating leaf chlorophyll content by measuring light absorption of specific spectral bands in living leaves (Watanabe et al. 1980; Chubachi et al. 1986). The methods for measurement of SPAD values are simple and quick, and close correlations between SPAD values and leaf photosynthesis values have been observed in rice (Huang and Peng 2004; Kato et al. 2004; Kumagai et al. 2009). Therefore, SPAD measurement may be a more appropriate method than 
destructive measurement of leaf chlorophyll content for use in genetic analysis of leaf photosynthesis.

Recent progress in the development of molecular markers has enabled the genetic mapping of quantitative trait loci (QTLs) for photosynthesis-related traits. Several putative QTLs have been detected for SPAD value or chlorophyll content in rice (Ishimaru et al. 2001; Teng et al. 2004; Abdelkhalik et al. 2005; Yue et al. 2006; Kanbe et al. 2008), and some of these have been confirmed by mapping in advanced-generation progeny (Kanbe et al. 2008). However, none of these QTLs has been precisely mapped as a Mendelian factor or characterized for its contribution to leaf photosynthesis.

In this study, we focused on the SPAD value of flag leaves at heading stage because higher leaf photosynthesis of flag leaves at heading stage may be critically related with high yield (Takai et al. 2006; Ohsumi et al. 2007). Then we identified a candidate QTL controlling SPAD value of flag leaves at heading stage by using backcross inbred lines (BILs) derived from a cross between japonica cultivar Sasanishiki and indica cultivar Habataki (Nagata et al. 2002). To confirm the putative QTL and to assess its association with leaf photosynthesis, we used chromosome segment substitution lines (CSSLs). In each of the CSSLs, a particular chromosome segment of Sasanishiki has been replaced by the corresponding segment from Habataki (Ando et al. 2008). Then, by using progeny derived from a cross between Sasanishiki and a CSSL harboring the target QTL, we conducted substitution mapping of the QTL. We also investigated the genetic relationship between SPAD value and specific leaf area (SLA), which is assumed to be correlated with leaf thickness. The QTL detected in this study appears to be associated with increased leaf photosynthetic rate and may also be associated with SLA.

\section{Results}

QTL detection in BILs and CSSLs

The mean SPAD value of flag leaves at heading stage was significantly greater in Habataki (44.4) than in Sasanishiki
(31.6) (Fig. 1). The SPAD values of the BILs ranged from 26.2 to 40.2 , all less than the value of Habataki. While there was only a 2-day difference in days-to-heading between Sasanishiki and Habataki (104 and 106 days, respectively), the BILs showed transgressive segregation (98-117 days).

QTL analysis of the BILs detected a large-effect QTL for SPAD value on the long arm of chromosome 4 (Fig. 2). The QTL explained $31.3 \%$ of total variance in the trait, and the Habataki allele increased the SPAD value (Table 1). Three QTLs for days-to-heading, each of which explained $9.8 \%$ to $16.3 \%$ of phenotypic variance $\left(R^{2}\right)$, were detected on chromosomes 7, 8, and 12. The Habataki alleles of the QTLs on chromosomes 7 and 12 and the Sasanishiki allele of the QTL on chromosome 8 increased days-to-heading. The map locations indicate that the QTL for SPAD value was not associated with a pleiotropic effect of a QTL for days-to-heading.

In the 39 CSSLs, SPAD values ranged from 28.9 to 35.4 units (Fig. 3a). The SPAD value of SL414 was significantly higher, by 3.4 points, than that of Sasanishiki, but also significant lower than that of Habataki. Phenotype and genotype data obtained for each CSSL confirmed that the region affecting SPAD value is located on the long arm of chromosome 4 (Fig. 3b). The candidate region was mapped to the interval between simple sequence repeat (SSR) markers RM3916 and RM2431.

\section{Leaf photosynthetic ability in SL414}

Because SL414 appeared to contain the QTL allele associated with increased SPAD value, we investigated leaf photosynthesis in SL414. We found significant differences in both leaf photosynthetic rate $\left(P_{\mathrm{n}}\right)$ and SPAD value among Sasanishiki, SL414, and Habataki (Fig. 4). SL414 had significantly higher $P_{\mathrm{n}}$ and SPAD values than Sasanishiki and significantly lower values than Habataki. Although stomatal conductance $\left(g_{\mathrm{s}}\right)$ did not differ significantly between Sasanishiki and SL414, SLA was significantly lower in SL414 than in Sasanishiki, implying that SL414 had thicker flag leaves than Sasanishiki. These results indicate the association among SPAD value, SLA, and $P_{n}$.
Fig. 1 Frequency distribution of SPAD value of flag leaves at heading and days-to-heading in 85 BILs derived from a cross of Sasanishiki $\times$ Habataki (Nagata et al. 2002). Vertical lines denote mean parental values; horizontal lines denote SD.
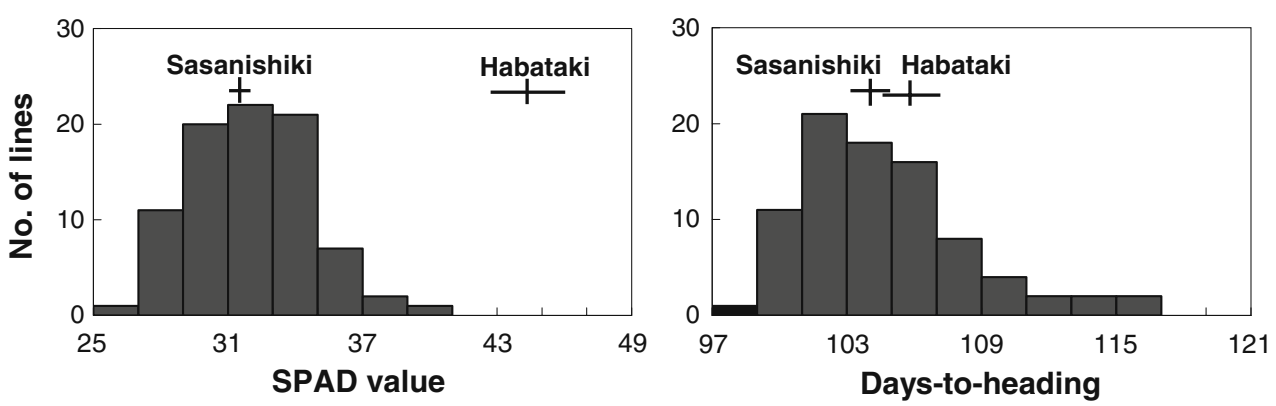


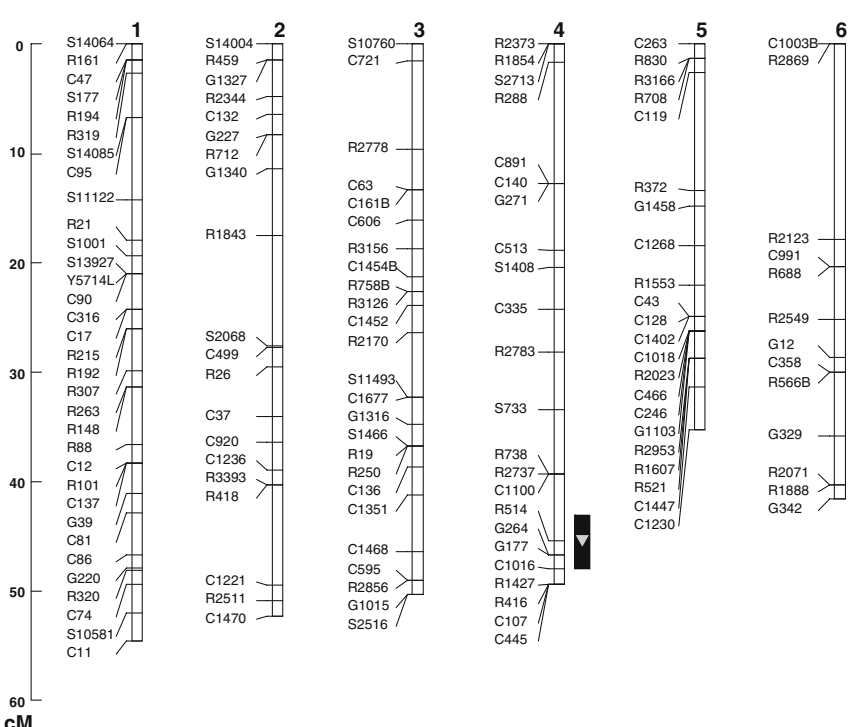

Fig. 2 Chromosomal locations of QTLs for days-to-heading and SPAD value of flag leaves at heading mapped in a set of 85 BILs (Nagata et al. 2002). Chromosome numbers are indicated above each linkage map. Marker names are located to the left of each linkage map. Triangles and boxes to the right of each linkage map represent LOD

Substitution mapping of the QTL for SPAD value

To verify the position of the QTL for SPAD value on the long arm of chromosome 4, we used $119 \mathrm{~F}_{2}$ progeny derived from a cross between SL414 and Sasanishiki. We detected a QTL near RM3534 that explained $68 \%$ of total phenotypic variance in SPAD value of flag leaves at heading stage (Table 2). The Habataki allele increased SPAD value by 2.3 units. A QTL for SLA was also detected close to RM3534, accounting for $30.7 \%$ of the total phenotypic variance. The Habataki allele decreased SLA by $11.1 \mathrm{~cm}^{2} \mathrm{~g}^{-1}$. We classified the $F_{2}$ progeny by the genotype of RM3534 (Fig. 5). $F_{2}$ plants homozygous for the Habataki allele had higher SPAD values and lower SLAs than those homozygous for the Sasanishiki allele.

Table 1 Putative QTLs Controlling SPAD Value of Flag Leaves at Heading Stage and Days-to-Heading in BILs between Sasanishiki and Habataki

\begin{tabular}{lllrrr}
\hline Trait & Chr. & Flanking marker & LOD & $A^{\mathrm{b}}$ & \multicolumn{1}{c}{$R^{2 \mathrm{c}}$} \\
\hline SPAD value & 4 & R514 & 9.1 & 2.2 & 31.3 \\
Days-to-heading & 7 & G1068 & 5.0 & 2.0 & 13.9 \\
& 8 & C1121 & 3.7 & -1.9 & 9.8 \\
& 12 & R367 & 5.4 & 2.1 & 16.3
\end{tabular}

\footnotetext{
${ }^{\mathrm{a}}$ The LOD peak of each QTL was at the position of the indicated DNA marker

${ }^{\mathrm{b}}$ Additive effect of the Habataki allele compared with the Sasanishiki allele

${ }^{\mathrm{c}}$ Percentage of phenotypic variance explained by each QTL
}

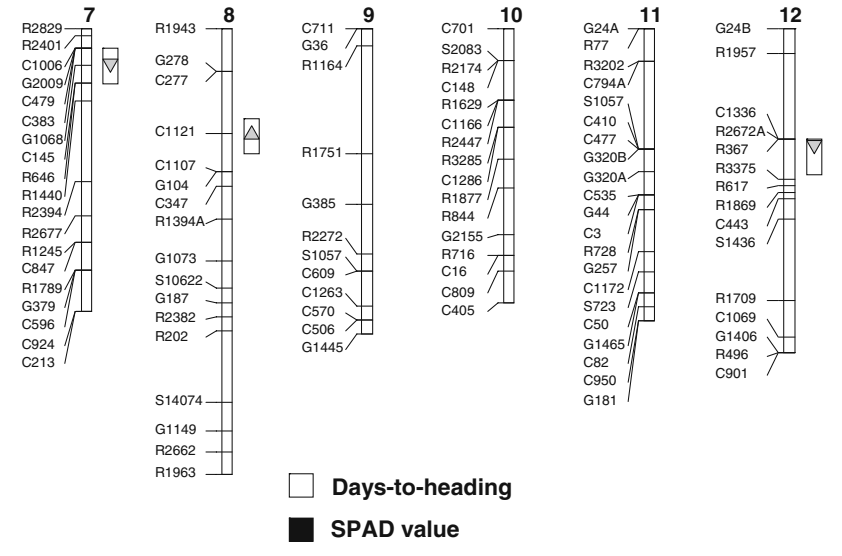

peaks of putative QTLs and their 1-LOD support intervals (van Ooijen 1992), respectively. Upward and downward triangles indicate that the trait value was increased by the Sasanishiki or Habataki allele, respectively.

The heterozygous plants were intermediate between the homozygotes in both traits. These results clearly confirm that the QTLs for SPAD value and SLA are located on the long arm of chromosome 4 and appear to be inherited as a single Mendelian factor.

To further delimit the candidate genomic region of the QTL for SPAD value, we genotyped $542 \mathrm{~F}_{2}$ plants derived from a cross between SL414 and Sasanishiki and identified 13 homozygous lines with recombination near RM3534 (Fig. 6). Four lines (lines 6-9) had significantly higher SPAD values than Sasanishiki, similar to the value of SL414. On the basis of the phenotype and genotype data, we delimited the candidate region of the QTL to a $1,798-\mathrm{kb}$ interval between RM5503 and RM17525. The SLA in lines 6-9 was also significantly lower than that in Sasanishiki. This indicates that the candidate genomic region of the QTL for SLA is also located between RM5503 and RM17525.

\section{Discussion}

Grain yield in cereals is determined by the balance between sink size and source capacity. The genetics of sink size (e.g., grain size and grain number) has been well analyzed in rice plants, and several QTLs controlling grain number per panicle and grain size have been identified (Ashikari et al. 2005; Fan et al. 2006; Song et al. 2007; Shomura et al. 2008; Huang et al. 2009). On the other hand, genetic analyses of factors affecting source capacity, such as photosynthetic rate, have been limited, probably because of the need for time- 
(a)

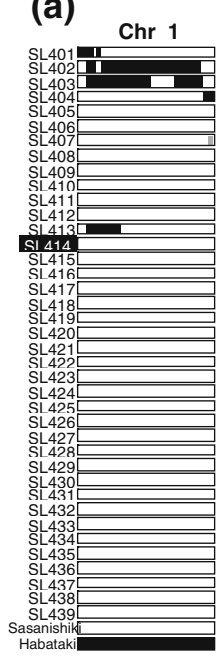

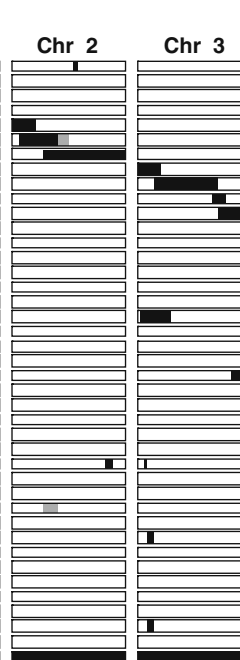

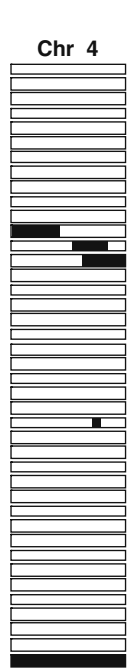

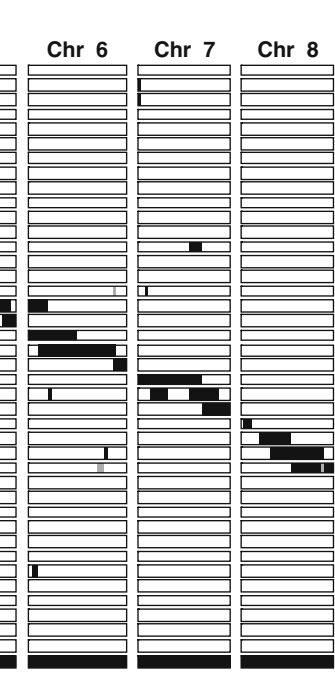
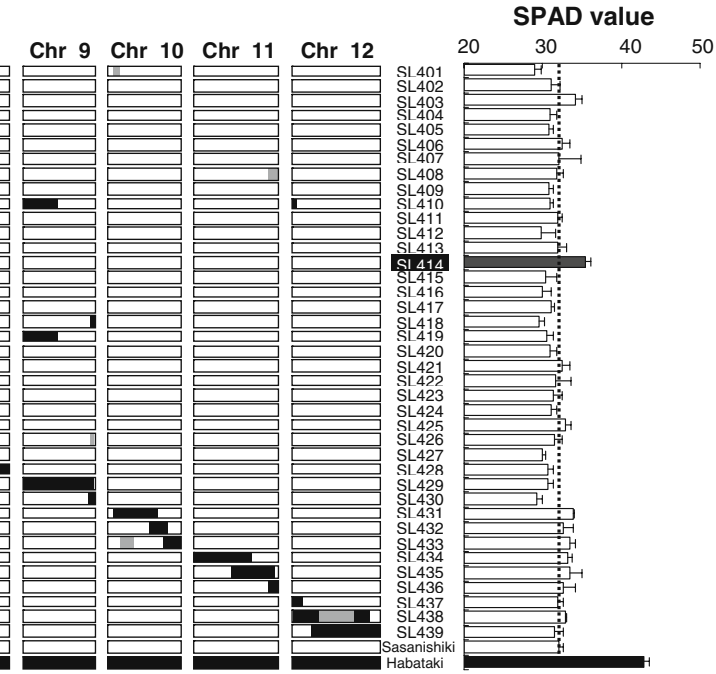

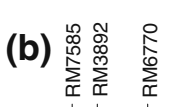

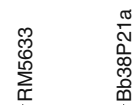

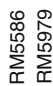

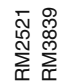

\section{1}
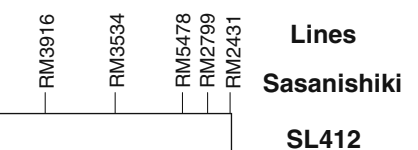

SPAD value

32.0

29.9

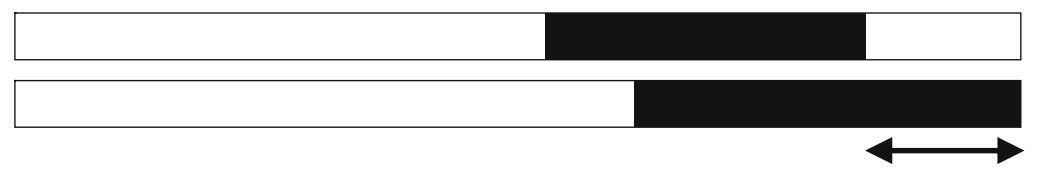

SL413

31.9

SL414

$35.4^{\star *}$

Fig. 3 Substitution mapping of QTLs affecting SPAD value in 39 CSSLs derived from a cross between Sasanishiki and Habataki. a (left) Graphical genotypes of the 39 CSSLs, Sasanishiki, and Habataki (Ando et al. 2008). Black denotes regions homozygous for Habataki alleles, white denotes regions homozygous for Sasanishiki alleles, and gray denotes heterozygous regions. (right) SPAD values corresponding to each graphical genotype. Bars indicate means, and error bars indicate $S D$.
Black indicates a significant difference from Sasanishiki at the $1 \%$ level by Dunnett's test. b Chromosome region affecting SPAD value (shown by double-headed arrow) on chromosome 4, predicted from the difference in SPAD values between each CSSL and Sasanishiki. **Significant difference from Sasanishiki at the 1\% level by Dunnett's test.
Fig. 4 Comparisons of photosynthesis-related traits of flag leaves at heading stage among Sasanishiki, SL414, and Habataki. $P_{\mathrm{n}}$, leaf photosynthetic rate; $g_{\mathrm{s}}$, stomatal conductance; SLA, specific leaf area. Bars indicate means and error bars indicate SD. Different letters show significant differences at the $1 \%$ level based on Tukey's test.
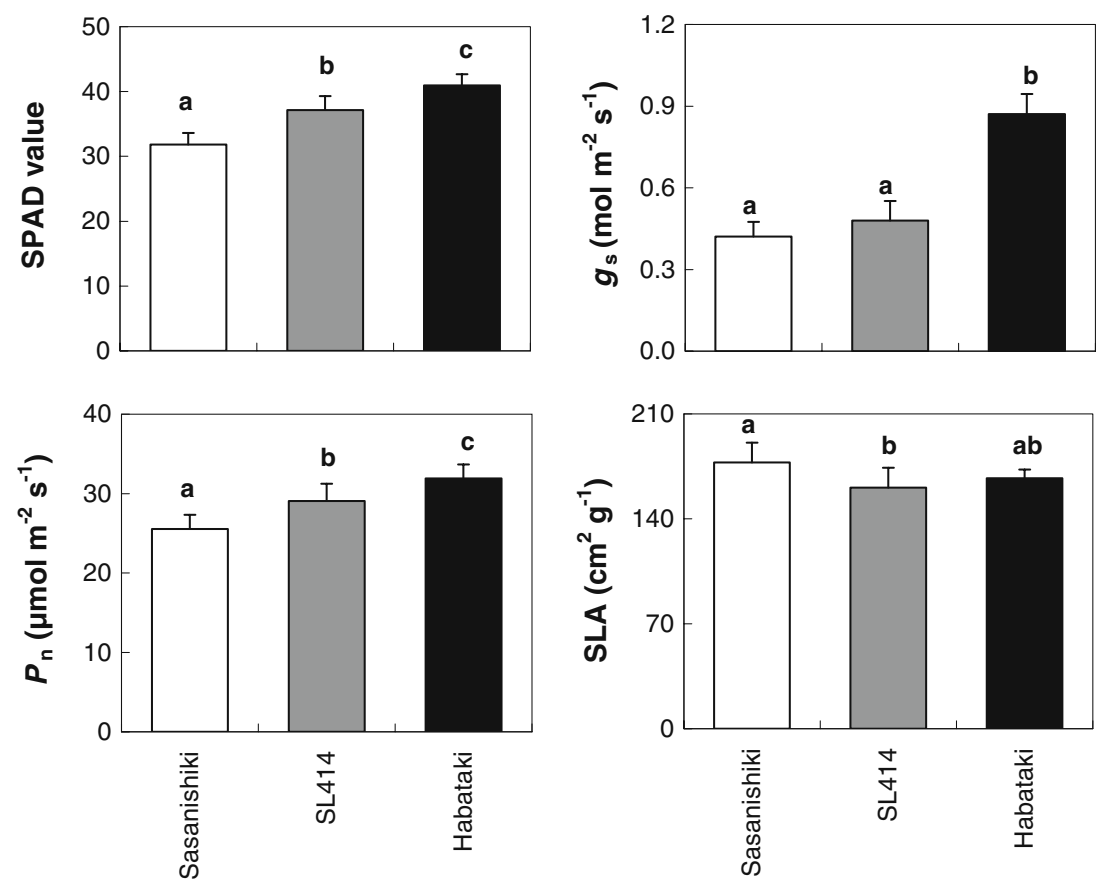
Table 2 Putative QTLs Controlling SPAD Value and SLA Detected in an $\mathrm{F}_{2}$ Population Derived from SL414×Sasanishiki

\begin{tabular}{|c|c|c|c|c|c|c|}
\hline Trait & Chr. & Flanking marker ${ }^{\mathrm{a}}$ & LOD & $A^{\mathrm{b}}$ & $D^{\mathrm{c}}$ & $R^{2 \mathrm{~d}}$ \\
\hline SPAD value & 4 & RM3534 & 29.9 & 2.3 & 0.3 & 68.0 \\
\hline SLA & 4 & RM3534 & 9.6 & -11.1 & -1.3 & 30.7 \\
\hline
\end{tabular}

${ }^{a}$ The LOD peak of each QTL was at the position of the indicated DNA marker

${ }^{\mathrm{b}}$ Additive effect of the Habataki allele compared with the Sasanishiki allele

${ }^{\mathrm{c}}$ Dominant effect of the Habataki allele compared with the Sasanishiki allele

${ }^{d}$ Percentage of phenotypic variance explained by QTL

consuming direct measurements, complex genetic control, and variability under various environmental conditions (Takai et al. 2009; Yamamoto et al. 2009). However, it is necessary to understand source ability in more detail to increase yield potential in rice. Therefore, we focused on chlorophyll content (SPAD value) as an index of leaf photosynthesis.

We detected a large-effect QTL $\left(R^{2}=31.3 \%\right)$ for SPAD value of flag leaves at heading stage on the long arm of chromosome 4 (Fig. 2). Since no other QTLs were detected in this population, the remaining $68.7 \%$ of phenotypic variance may be due to environmental factors, measuring error or false negative QTL with minor effect. Previous studies have also detected QTLs for chlorophyll content in this region (Yue et al. 2006; Kanbe et al. 2008). The positions of markers flanking these QTLs were similar
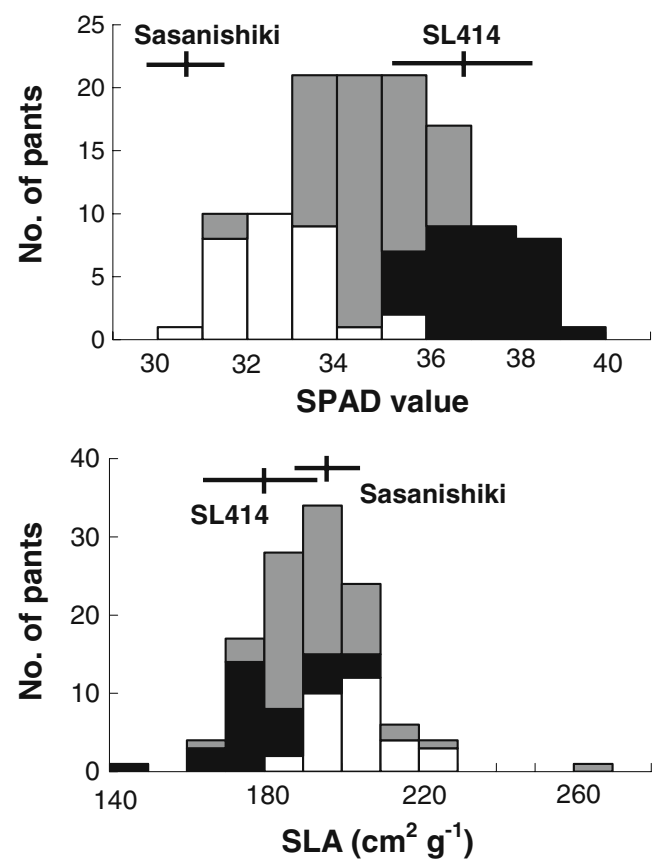

Fig. 5 Frequency distribution of SPAD values and SLAs of flag leaves at heading in $119 \mathrm{~F}_{2}$ plants derived from a cross between SL414 and Sasanishiki, classified by the genotype for marker RM3534. White indicates plants homozygous for the Sasanishiki allele, black indicates plants homozygous for the Habataki allele, and gray indicates heterozygous plants. among these studies, so the QTL detected here may be same as those identified previously. Besides, the map location of the QTL for SPAD value was different from those of QTLs for days-to-heading, indicating the QTL for SPAD value was not associated with a pleiotropic effect of a QTL for days-to-heading.

We confirmed the QTL identified in this study by substitution mapping in a set of CSSLs (Fig. 3). CSSLs are useful to characterize and detect QTLs because phenotypic differences can be evaluated within a uniform genetic background (Ebitani et al. 2005; Yamamoto et al. 2009). One of the CSSLs, SL414, contained a Habataki chromosome segment on the long arm of chromosome 4 and had significantly higher $P_{\mathrm{n}}$ and SPAD values than Sasanishiki (Fig. 4). The values of both traits in SL414 were intermediate between Sasanishiki and Habataki. These results indicate that the segment harboring the QTL for SPAD value was also highly associated with increased $P_{\mathrm{n}}$.

In general, leaf photosynthesis by $\mathrm{C}_{3}$ crops is determined by both the $\mathrm{CO}_{2}$ supply obtained through stomata and the fixation of $\mathrm{CO}_{2}$ in the chloroplasts (Farquhar and Sharkey 1982). Our study did not detect any difference in $g_{\mathrm{s}}$ between SL414 and Sasanishiki (Fig. 4), which indicates that the QTL detected here is involved in $\mathrm{CO}_{2}$ fixation rather than in $\mathrm{CO}_{2}$ supply. Rather, the difference in SPAD value between SL414 and Sasanishiki reflects a difference in chlorophyll content or leaf $\mathrm{N}$ content per unit leaf area. Higher chlorophyll content per unit leaf area may reflect the presence of a larger number of chloroplasts per mesophyll cell and/or higher chlorophyll content per chloroplast in cases where leaf thicknesses do not differ. However, previous studies have indicated that SPAD values may sometimes reflect variation in leaf thickness, because the readings are based on the leaf chlorophyll's absorption of specific spectral bands of light, which may be influenced by leaf thickness (Peng et al. 1993; Jinwen et al. 2009). The SLA, which is assumed to be correlated with leaf thickness, was significantly lower in SL414 than in Sasanihiki, and similar between SL414 and Habataki (Fig. 4). These results suggest that the higher SPAD value of SL414 resulted from thicker leaves. Thicker leaves are considered to be important for increasing leaf photosynthesis because they 


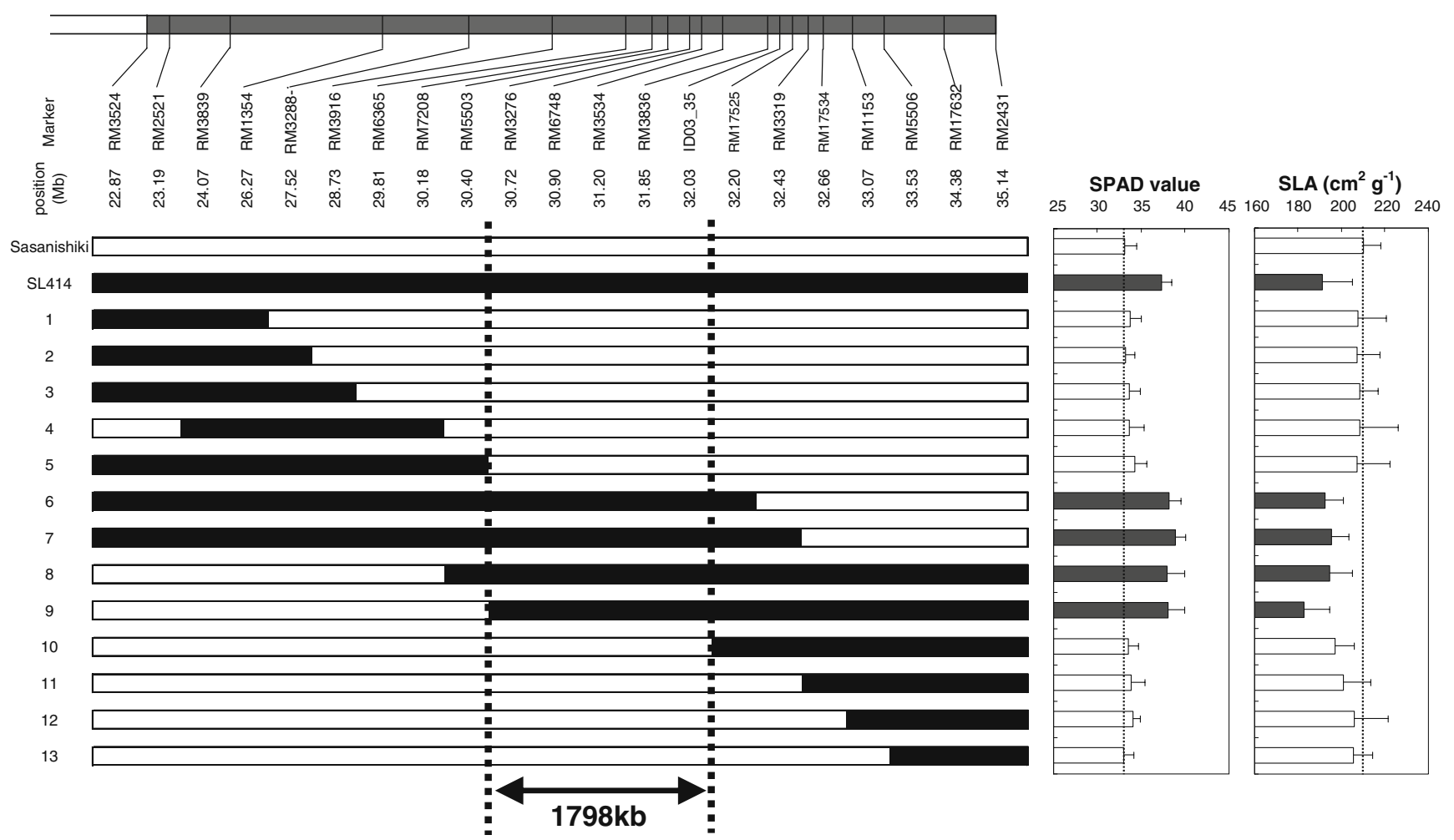

Fig. 6 Substitution mapping of QTLs controlling SPAD value and SLA of flag leaves at heading stage on the long arm of chromosome 4 based on $13 \mathrm{~F}_{3}$-derived lines. (left) Graphical genotypes. Black denotes regions homozygous for Habataki alleles; white denotes regions homozygous for Sasanishiki alleles. The candidate QTL

can capture light energy efficiently by more chlorophyll per unit leaf area and they are better able to protect the area where the chloroplast surface faces intercellular spaces, allowing more efficient $\mathrm{CO}_{2}$ diffusion and transport (Terashima et al. 2006). Higher leaf $\mathrm{N}$ content per unit leaf area and higher $g_{s}$ (seen in Habataki compared with Sasanishiki) are believed to be important factors contributing to varietal differences in leaf photosynthesis (Asanuma et al. 2008; Takai et al. 2010). Since measurements taken on a unit leaf area basis are expected to be influenced by leaf thickness, the higher $\mathrm{N}$ content per unit leaf area in Habataki might be also caused by thicker leaves. To verify either leaf thickness is associated with the QTL for SPAD value, it is necessary to conduct further in-depth studies such as spectrophotometric chlorophyll measurement and comparison of the cross sections of leaf blade.

Using advanced-generation progeny derived from a cross between SL414 and Sasanishiki, we confirmed the QTL for SPAD value and delimited the candidate region to a 1798-kb interval between RM5503 and RM17525 (Fig. 6). Although a mutant gene associated with chlorophyll content, $G c$, was recently mapped to chromosome 1 (Wang et al. 2008), no QTLs for SPAD value or chlorophyll content have previously been delimited; this is the first study region is indicated by a double-headed arrow. (right) SPAD values and SLA values. Bars indicate means and error bars indicate SD. Black indicates a significant difference from Sasanishiki at the $1 \%$ level by Dunnett's test.

in rice to identify a QTL involving a leaf photosynthesisrelated trait.

It is of interest that the QTL for SLA was also delimited to the same region as the QTL for SPAD value. This result strongly suggests that the QTLs for SPAD value and SLA are associated with either the pleiotropic effects of a single QTL or the effects of two tightly linked loci. These results also indicate that the QTLs may play an important role in increasing leaf photosynthesis by increasing chlorophyll content or leaf thickening, or both. To determine whether pleiotropy or tight linkage is responsible for the apparent proximity of these QTLs, and to evaluate the specific contribution of the QTLs to leaf photosynthesis in rice plants, we are now working to clone the two QTLs. Moreover, cloning of the QTLs and development of NILs will help to elucidate whether an increase in leaf photosynthesis could contribute to yield improvement. Because leaf photosynthesis is one of the components of canopy photosynthesis associated with biomass production and yield (Peng 2000; Yoshida and Horie 2009), higher leaf photosynthesis would be expected to increase final yield, unless other components such as leaf area change. Our study suggests two possible means to increase leaf photosynthesis: morphological modification of leaves (e.g., 
thicker leaves) and physiological modification of leaves (e.g., higher chlorophyll content). A significant challenge to overcome is that morphological modifications such as thicker leaves might be accompanied by reductions in leaf area. Further studies are necessary to elucidate which modifications of leaf photosynthesis could improve yield.

\section{Materials and methods}

Plant materials and cultivation

Two cultivars, Sasanishiki (japonica) and Habataki (indica), were used in this study. Habataki is a high-yielding cultivar from Japan (Kobayashi et al. 1990) with a greater photosynthetic rate in the flag leaves at heading stage than Sasanishiki (Takai et al. 2010).

We used 85 BILs (Nagata et al. 2002) and 39 CSSLs (Ando et al. 2008) derived from a cross between Sasanishiki and Habataki for the QTL analysis. Rice plants were grown in a paddy field at National Institute of Agrobiological Sciences (NIAS) in Tsukuba, Japan, in 2007. Thirty-day-old seedlings of each line were transplanted at one seedling per hill on 16 May. Each line was planted in a single row of 12 hills at a spacing of $15 \mathrm{~cm}$ between hills and $30 \mathrm{~cm}$ between rows. Basal fertilizer was applied: $56 \mathrm{~kg} \mathrm{~N}, 56 \mathrm{~kg} \mathrm{P}$, and $56 \mathrm{~kg} \mathrm{~K} \mathrm{ha}{ }^{-1}$. Additional $\mathrm{N}$ fertilizer was top-dressed at $30 \mathrm{~kg} \mathrm{~N} \mathrm{ha}^{-1} 2$ weeks after transplanting. Three plants per line were selected for the measurement of SPAD value.

On the basis of initial results, we performed additional analyses using SL414, a Sasanishiki-derived CSSL in which part of the long arm of chromosome 4 is substituted with the corresponding segment from Habataki. SL414 was crossed with Sasanishiki, and 119 self-pollinated $\mathrm{F}_{2}$ progeny and the parents were raised in the NIAS paddy field for traits investigation in 2008. Thirty-day-old seedlings were transplanted into the field on 4 June. Plant density and fertilizer treatment were the same as in 2007. Each $F_{2}$ plant was used for the measurement of SPAD value. For substitution mapping of the candidate QTL, additional $423 \mathrm{~F}_{2}$ seeds were sown in a growth chamber room, and we used DNA markers to identify 13 out of 542 $(119+423) \mathrm{F}_{2}$ plants with recombination near the QTL, and harvested $F_{3}$ seeds. From each of the $13 \mathrm{~F}_{3}$ lines, we selected one $\mathrm{F}_{3}$ plant that was homozygous for the recombinant chromosome identified in the $\mathrm{F}_{2}$ parent in the growth chamber during the winter season in 2008. The $F_{3}$ plants were self-pollinated, producing $13 \mathrm{~F}_{4}$ lines that were used for substitution mapping of the target QTL. $\mathrm{F}_{4}$ plants were grown in a randomized complete block design with three replications in a paddy field at the National Institute of Crop Science in Miraidaira, Japan, in 2009. Twenty-oneday-old seedlings were transplanted at one seedling per hill on 4 June. Each plot consisted of one row with 15 hills. The plant density was the same as in 2007. Basal fertilizer was applied: $60 \mathrm{~kg} \mathrm{~N}, 52 \mathrm{~kg} \mathrm{P}$, and $75 \mathrm{~kg} \mathrm{~K} \mathrm{ha}^{-1}$. Fifteen plants per line (five in each plot) were selected for the measurement of SPAD value.

\section{Phenotypic measurements}

At heading stage, determined as the number of days from sowing to heading of the first panicle (days-to-heading) in five plants for each BIL and CSSL, the SPAD value of the fully extended flag leaf on the main stem was measured with a SPAD meter (SPAD-502, Konica-Minolta, Japan). Three out of five plants investigated for days-to-heading were used for the measurement of SPAD value for each BIL and CSSL. Six readings around the middle of each leaf blade were averaged. In 2008 and 2009, for Sasanishiki, Habataki, SL414, and SL414 progeny, SLA of flag leaves used for SPAD measurement was calculated as the ratio of leaf area to leaf dry weight; lower SLA values indicated thicker leaves. Digital images of the flag leaves were used for measurement of leaf area with computer software (LIA32, Nagoya University, Japan). Leaf dry weight was determined after oven-drying.

In 2008, we measured the photosynthetic rate of Sasanishiki, Habataki, and SL414 flag leaves at heading stage with a portable photosynthesis system (LI-6400, Li-Cor, Lincoln, NE, USA). Measurement was conducted on clear days between 0900 and $1300 \mathrm{~h}$ under a constant saturated light level of $2,000 \mu \mathrm{mol} \mathrm{m}^{-2} \mathrm{~s}^{-1}$ provided by red/ blue light-emitting diodes. The leaf chamber temperature was maintained at $30^{\circ} \mathrm{C}$, the reference $\mathrm{CO}_{2}$ concentration was $380 \mu \mathrm{mol} \mathrm{mol}^{-1}$, and the relative humidity was $75 \% \pm$ $5 \%$. Gas-exchange parameters were recorded once the topmost expanded leaf was enclosed in the chamber and the system software indicated that $\mathrm{CO}_{2}, \mathrm{H}_{2} \mathrm{O}$, and flow in the chamber were stabilized. One flag leaf from each of ten different plants per cultivar or line was measured.

\section{QTL analysis}

For QTL analysis of BILs and substitution mapping of CSSLs, 236 RFLP markers (Nagata et al. 2002) and 166 PCR-based markers (Ando et al. 2008), respectively, were used. An additional ten SSR markers developed by McCouch et al. (2002) and the International Rice Genome Sequencing Project (2005) were used for genotyping SL414 $\times$ Sasanishiki $F_{2}$ plants. We used four SSR markers and one insertion-deletion (InDel) marker to determine genotypes of $13 \mathrm{~F}_{3}$-derived lines for substitution mapping of the target QTL. The InDel marker ID03_35 was constructed by using sequence information in the rice DNA polymorphism database (Shen et al. 2004). The 
sequences of the forward and reverse primers were 5'GCTCCGGTGGCTCTTCGTG-3' and 5'-AGGCTTAAG GCGAAAGGAAGT-3', respectively. Total DNA of each plant was extracted from leaves by the CTAB method (Murray and Thomson 1980). Linkage maps were constructed in MAPMAKER/EXP 3.0 software (Lander et al. 1987). The chromosomal positions and effects of putative QTLs were determined by composite interval mapping in QTL Cartographer 2.0 software (Basten et al. 2002). The threshold of QTL detection was based on 1,000 permutation tests at the $5 \%$ level of significance (Churchill and Doerge 1994; Doerge and Churchill 1996). The additive and dominant effects and phenotypic variance explained by each QTL $\left(R^{2}\right)$ were estimated from the peak LOD score. For substitution mapping of CSSLs, the significance of the difference in SPAD value between Sasanishiki and each CSSL was determined by Dunnett's test (JMP 6.0.3 software, SAS Institute, Cary, NC, USA).

Acknowledgments We thank the staff of the technical support section of NIAS and NICS for field management. This work was supported by a grant from the Ministry of Agriculture, Forestry and Fisheries of Japan (Genomics for Agricultural Innovation, QTL1002).

\section{References}

Abdelkhalik AF, Shishido R, Nomura K, Ikeshashi H. QTL-based analysis of leaf senescence in an indica/japonica hybrid in rice (Oryza sativa L.). Theor Appl Genet. 2005;110:1226-35.

Ando T, Yamamoto T, Shimizu T, Ma XF, Shomura A, Takeuchi Y, et al. Genetic dissection and pyramiding of quantitative traits for panicle architecture by using chromosomal segment substitution lines in rice. Theor Appl Genet. 2008;116:881-90.

Asanuma S, Tsuru Y, Nito N, Ookawa T, Hirasawa T. Factors affecting the differences in the maximum rate of photosynthesis measured by gas exchange method between rice cultivars Sasanishiki and Habataki. Jpn J Crop Sci. 2008;77(2):118-9.

Ashikari M, Sakakibara H, Lin S, Yamamoto T, Takashi T, Nishimura $\mathrm{A}$, et al. Cytokinin oxidase regulates rice grain production. Science. 2005;309:741-5.

Basten CJ, Weir BS, Zeng ZB. A reference manual and tutorial for QTL mapping, QTL cartographer. Version 1.16. Raleigh: North Carolina State University; 2002.

Chubachi T, Asano I, Oikawa T. The diagnosis of nitrogen nutrition of rice plants (Sasanishiki) using chlorophyll meter. Jpn J Soil Sci Plant Nutr. 1986;57:190-3.

Churchill GA, Doerge RW. Empirical threshold values for quantitative trait mapping. Genetics. 1994;138:963-71.

Doerge RW, Churchill GA. Permutation tests for multiple loci affecting a quantitative character. Genetics. 1996;142:285-94.

Ebitani T, Takeuchi Y, Nonoue Y, Yamamoto T, Takeuchi K, Yano M. Construction and evaluation of chromosome segment substitution lines carrying overlapping chromosome segments of indica rice cultivar 'Kasalath' in a genetic background of japonica elite cultivar 'Koshihikari'. Breed Sci. 2005;55:65-73.

Evans LT. Crop evolution, adaptation and yield. New York: Cambridge University; 1993.
Fan C, Xing Y, Mao H, Lu T, Han B, Xu C, et al. GS3, a major QTL for grain length and weight and minor QTL for grain width and thickness in rice, encodes a putative transmembrane protein. Theor Appl Genet. 2006;112:1164-71.

Farquhar GD, Sharkey TD. Stomatal conductance and photosynthesis. Ann Rev Plant Physiol. 1982;33:317-45.

Horie T, Matsuura S, Takai T, Kuwasaki K, Ohsumi A, Shiraiwa T. Genotypic difference in canopy diffusive conductance measured by a new remote-sensing method and its association with the difference in rice yield potential. Plant Cell Environ. 2006;29:653-60.

Huang J, Peng S. Comparison and standardization among chlorophyll meters in their readings on rice leaves. Plant Prod Sci. 2004;7:97-100.

Huang X, Qian Q, Liu Z, Sun H, He S, Luo D, et al. Natural variation at the DEP1 locus enhances grain yield in rice. Nat Genet. 2009;41:494-7.

Hubbart S, Peng S, Horton H, Chen Y, Murchie EH. Trends in leaf photosynthesis in historical rice varieties developed in the Philippines since 1966. J Exp Bot. 2007;58:3429-38.

International Rice Genome Sequencing Project. The map-based sequence of the rice genome. Nature. 2005;436:793-800.

Ishimaru K, Yano M, Aoki N, Ono K, Hirose T, Lin SY, et al. Toward the mapping of physiological and agronomic characters on a rice function map: QTL analysis and comparison between QTLs and expressed sequence tags. Theor Appl Genet. 2001;102:793-800.

Jinwen L, Jingping Y, Pinpin F, Junlan S, Dongsheng L, Changshui G, et al. Responses of rice leaf thickness, SPAD readings and chlorophyll $\mathrm{a} / \mathrm{b}$ ratios to different nitrogen supply rates in paddy field. Field Crops Res. 2009;114:426-32.

Kanbe T, Sasaki H, Aoki N, Yamagishi T, Ebitani T, Yano M, et al. Identification of QTLs for improvement of plant type in rice (Oryza sativa L.) Using Koshihikari/Kasalath chromosome segment substitution lines and backcross progeny F2 population. Plant Prod Sci. 2008;11:447-556.

Kato M, Kobayashi K, Ogiso E, Yokoo M. Photosynthesis and drymatter production during ripening stage in a female-sterile line of rice. Plant Prod Sci. 2004;7:184-8.

Kobayashi A, Koga Y, Uchiyamada H, Horiuchi H, Miura K, Okuno $\mathrm{K}$, et al. Breeding a new rice variety "Habataki". Bull Hokuriku Natl Agric Exp Stn. 1990;32:65-84.

Kumagai E, Araki T, Kubota F. Correlation of chlorophyll meter readings with gas exchange and chlorophyll fluorescence in flag leaves of rice (Oryza sativa L.) plants. Plant Prod Sci. 2009;12:50-3.

Kura-Hotta M, Satoh K, Katoh S. Relationship between photosynthesis and chlorophyll content during leaf senescence. Plant Cell Physiol. 1987;28:1321-9.

Lander ES, Green P, Abrahamson J, Barlow A, Daley MJ, Lincoln SE, et al. MAPMAKER: an interactive computer package for constructing primary genetic linkage maps of experimental and natural populations. Genomics. 1987;1:174-81.

Makino A, Mae T, Ohira K. Photosynthesis and ribulose 1, 5bisphosphate carboxylase in rice leaves: changes in photosynthesis and enzymes involved in carbon assimilation from leaf development through senescence. Plant Physiol. 1983;73:1002-7.

McCouch SR, Teytelman L, Xu Y, Lobos KB, Clare K, Walton M, et al. Development and mapping of 2240 new SSR markers for rice (Oryza sativa L.). DNA Res. 2002;9:199-207.

Murray MG, Thomson WF. Rapid isolation of high molecular weight plant DNA. Nucleic Acids Res. 1980;8:4321-6.

Nagata K, Fukuta Y, Shimizu S, Yagi T, Terao T. Quantitative trait loci for sink size and ripening traits in rice (Oryza sativa L.). Breed Sci. 2002;52:259-73.

Ohsumi A, Hamasaki A, Nakagawa H, Yoshida H, Shiraiwa T, Horie T. A model explaining genotypic and ontogenetic variation of 
leaf photosynthetic rate in rice (Oryza sativa) based on leaf nitrogen content and stomatal conductance. Ann Bot London. 2007;99:265-73.

Peng S. Single-leaf and canopy photosynthesis of rice. In: Sheehy JE, Mitchell PL, Hardy B, editors. Redesigning rice photosynthesis to increase yield. Philippines: International Rice Research Institute; 2000. p. 213-28.

Peng S, Garcia FV, Laza RC, Cassman KG. Adjustment for specific leaf weight improves chlorophyll meter's estimate of rice leaf nitrogen concentration. Field Crops Res. 1993;85:987-90.

Peng S, Khush GS, Virk P, Tang Q, Zou Y. Progress in ideotype breeding to increase yield potential. Field Crops Res. 2008;108:32-8.

Porra RJ, Thompson WA, Kriedemann PE. Determination of accurate extinction coefficients and simultaneous equations for assaying chlorophylls $\mathrm{a}$ and $\mathrm{b}$ extracted with four different solvents: verification of the concentration of chlorophyll standards by atomic absorption spectroscopy. Biochim Biophys Acta. 1989;975:384-94.

Shen YJ, Jiang H, Jin JP, Zhang ZB, Xi B, He YY, et al. Development of genome-wide DNA polymorphism database for map-based cloning of rice genes. Plant Physiol. 2004;135:1198-205.

Shomura A, Izawa T, Ebana K, Ebitani T, Kanegae H, Konishi S, et al. Deletion in a gene associated with grain size increased yields during rice domestication. Nat Genet. 2008;40:1023-8.

Sinclair TR, Purcell LC, Sneller CH. Crop transformation and the challenge to increase yield potential. Trends Plant Sci. 2004;9:70-5.

Song XJ, Huang W, Shi M, Zhu MZ, Lin HX. A QTL for rice grain width and weight encodes a previously unknown RING-type E3 ubiquitin ligase. Nat Genet. 2007;39:623-30.

Takai T, Matsuura S, Nishio T, Ohsumi A, Shiraiwa T, Horie T. Rice yield potential is closely related to crop growth rate during late reproductive period. Field Crops Res. 2006;96:328-35.

Takai T, Ohsumi A, San-oh Y, Laza MRC, Kondo M, Yamamoto T, et al. Detection of a quantitative trait locus controlling carbon isotope discrimination and its contribution to stomatal conductance in japonica rice. Theor Appl Genet. 2009;118:1401-10.

Takai T, Yano M, Yamamoto T. Canopy temperature on clear and cloudy days can be used to estimate varietal differences in stomatal conductance in rice. Field Crops Res. 2010;115:165-70.

Teng S, Qian Q, Zeng D, Kunihiro Y, Fujimoto K, Huang D, et al. QTL analysis of leaf photosynthetic rate and related physiological traits in rice (Oryza sativa L.). Euphytica. 2004;135:1-7.

Terashima I, Hanba YT, Tazoe Y, Vyas P, Yano S. Irradiance and phenotype: comparative eco-development of sun and shade leaves in relation to photosynthetic $\mathrm{CO}_{2}$ diffusion. J Exp Bot. 2006;57:343-54.

van Ooijen JW. Accuracy of mapping quantitative trait loci in autogamous species. Theor Appl Genet. 1992;84:803-11.

Wang F, Wang G, Li X, Huang J, Zheng J. Heredity, physiology and mapping of a chlorophyll content gene of rice (Oryza sativa L.). J Plant Physiol. 2008;165:324-30.

Watanabe SY, Hatanaka Y, Inada K. Development of a digital chlorophyll meter: I. Structure and performance. Jpn J Crop Sci. 1980;49(special issue):89.

$\mathrm{Xu}$ YF, Ookawa T, Ishihara K. Analysis of the photosynthetic characteristics of the high-yielding rice cultivar Takanari. Jpn J Crop Sci. 1997;66:616-23.

Yamamoto T, Yonemaru J, Yano M. Towards the understanding of complex traits: substantially or superficially? DNA Res. 2009;16:141-54.

Yoshida $\mathrm{H}$, Horie T. A process model for explaining genotypic and environmental variation in growth and yield of rice based on measured plant $\mathrm{N}$ accumulation. Field Crops Res. 2009;113:227-37.

Yue B, Xue WY, Luo LJ, Xing YZ. QTL analysis for flag leaf characteristics and their relationships with yield and yield traits in rice. Acta Genetica Sinica. 2006;33:824-32.

Zelitch I. The close relationship between net photosynthesis and crop yield. Bioscience. 1982;32:796-802.

Long SP, Zhu XG, Naidu SL, Ort DR. Can improvement in photosynthesis increase crop yields? Plant Cell Environ. 2006;29:315-30. 